\title{
Emotional Drain in Digital Design for Inheritance of Intangible Cultural Heritage
}

\author{
Ding Fanzhuo ${ }^{1 a}$, Ding Baihao ${ }^{2 b}$, Bai Yang ${ }^{3 \mathrm{c}}$, Zhang Jixiao ${ }^{4 \mathrm{~d}^{*}}$ \\ ${ }^{1}$ Beijing Forestry University College of Arts and Design, Beijing China. \\ ${ }^{2}$ Beijing Forestry University College of Arts and Design, Beijing China. \\ ${ }^{3}$ Beijing Forestry University College of Arts and Design, Beijing China. \\ ${ }^{4}$ Beijing Forestry University College of Arts and Design, Beijing China.
}

\begin{abstract}
As technology advances, digitalization has become more and more widespread. This is true of the continuation and inheritance of intangible cultural heritage in which digital technology is also increasingly utilized. For one thing, the contents of intangible cultural heritage that are difficult to preserve need to be refined for technological presentation. For another, the emotional connotation of intangible cultural heritage implied in the inheriting process should be retained as far as possible. This paper discusses that how digital technology broadens the path of inheriting intangible cultural heritage, analyzes the logical connection between the protection of intangible cultural heritage and digital technology, and also explores the future of developing intangible cultural heritage with the help of digital technology.
\end{abstract}

\section{INTRODUCTION}

Intangible cultural heritage is an indispensable cultural treasure of a nation and the world, and also an objective manifestation of the uniqueness and regional difference of a culture. With the rapid development of society, the protection of intangible cultural heritage and the inheritance of regional splendid culture have become the important part of culture development [1]. However, intangible cultural heritage has suffered continuous drain due to the double impact of vertical changes of the times and horizontal cultural exchanges [2]. In recent years, most of the mainstream means to "activate" intangible cultural heritage at home and abroad are technological means by which complex contexts of intangible cultural heritage are digitally transformed. Intangible cultural heritage can be well supported in terms of conservation and spread during the digitizing process of replacing physical communication with technological means, but in a sense such a process puts an end to the direct contact between people and intangible cultural heritage since the participants are essentially in contact with technology and cut off from the most direct experience and feeling, thus leading to the drain of emotion conveyed by intangible cultural heritage.

\section{PROBLEMS OF INHERITANCE OF INTANGIBLE Cultural Heritage}

\subsection{Aging Trend of Inheritance}

With the development of the information society, the intangible cultural heritage in the era of agricultural civilization has gradually deviated from the mainstream aesthetic, and thus is facing a crisis of survival [3]. The inheritance of intangible cultural heritage is faced with low public awareness and a particularly weak affinity among young people. With regard to the mode of transmission, it is not only necessary to make good use of the direct transmission from the elderly to the younger generation, but also to utilize interactive digital communication featured by the extensive spread range and massive information of the Internet [4]. Currently, most of the products made by using the techniques of intangible cultural heritage are traditional handicrafts, and some of them are improved and innovated by craftsmen in the production process. Although these products display rich regional characteristics and profound historical connotation, it is difficult for them to grow in young people's lives. This is because traditional products of intangible cultural heritage lack something interesting and playable and at the same time, their colors and decorative patterns differ from Today's popular forms, which makes it hard to meet the young people's need for the aesthetic in the new era and the pursuit of

\footnotetext{
ae-mail address: 535625995@qq.com

be-mail address: 469954273@qq.com

ce-mail address: 982415014@qq.com

${ }^{*}$ Corresponding author e-mail address: jixiao_zhang@163.com
} 
individuality. In the Convention for the Safeguarding of Intangible Cultural Heritage, it is stated that "[t]his intangible cultural heritage, transmitted from generation to generation, is constantly recreated by communities and groups in response to their environment, their interaction with nature and their history, and provides them with a sense of identity and continuity... [5]" Therefore, while retaining the original characteristic elements and cultural connotations, the products of intangible cultural heritage need to be designed and transformed in accordance with the trend of the times instead of blindly sticking to tradition. The brand-new innovation trend of intangible cultural heritage has come.

\subsection{Incomplete Data Collection}

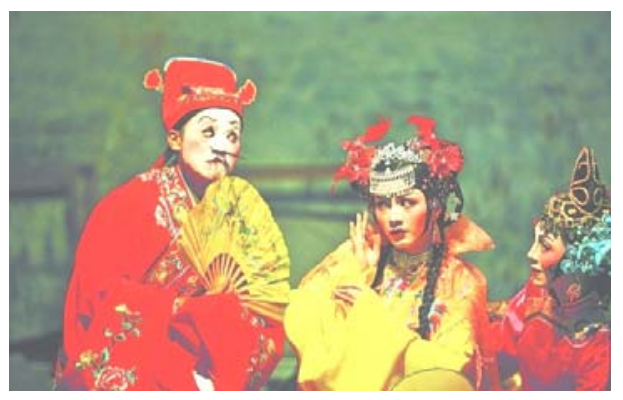

Figure 1. source: Southeast Express

In the era of big data, there are a large number of multi-dimensional information data based on streaming media and texts. They are an important part of today's network data, among which the number of intangible cultural heritage data is also rising rapidly. However, at present, the development of big data storage technology in China is still basically in the initial stage. Some technologically underdeveloped regions have not applied information technology to big data storage well, and lots of information and pictures relevant to intangible cultural heritage can hardly be searched out. The amount of existing intangible cultural heritage resources recorded in the network database accounts for a very small proportion in the total amount of intangible cultural heritage. Most of them are only spread to relevant regions or provinces, and further they are passed on orally, without systematic database registration. Even the items that have been included are not systematic, complete and specific, resulting in a very limited cognitive source of intangible cultural heritage for the public. For instance, the intangible cultural heritage Gaojia Opera in Southern Fujian (Figure 1) is in fact very influential in Southern Fujian and the countries along the Maritime Silk Road. It was once incorporated into the Chinese drama history in Southeast Asia. Its cultural impact can be imaged. But across the country, except for experts and scholars and the older generation in Fujian and Guangdong who enjoy listening to operas, perhaps, few Chinese people can describe the specific artistic paradigm and performance features of Gaojia Opera. As a result, it is necessary to make full use of the technological advantages of big data to obtain the important value of Internet-based big data mode in intangible cultural heritage information storage.

\section{VARIOUS POSSIBILITIES FOR INTANGIBLE Cultural Heritage Brought by Modern TECHNOLOGY}

\subsection{Reproducing Intangible Cultural Heritage by Digital Technology}

At present, there are some problems in the development, utilization and protection of intangible cultural heritage in China such as single protection, ineffective transmission and insufficient participation of the whole society. However, using digital technology in the protection of intangible cultural heritage can improve the realistic dilemma of intangible cultural heritage, that is, "intangible, can not experience". With the continuous improvement of technological level, the use of digital platform for data protection and storage has been widely recognized, and digital tools such as the establishment of cloud archives on big data platform, immersion modeling with virtual reality technology, and restoration through $3 \mathrm{D}$ printing technology have become the most fundamental methods. Take the medieval Romanesque paintings of the Pyrenees in Catalonia (Figure. 2) as an example. Thanks to the use of digital mapping techniques, many such works of art that have been eroded because of their long history have now been restored [6]. Currently, China's most frequently used digital technologies mainly focus on data retention technology with graphics and images, spatial data collection and storage as the core, information processing technology based on three-dimensional modeling and cultural creativity, as well as transmission technology with virtual reality and network display as the core [7]. Among them, the digital media based on mixed reality technology (VR) can not only enhance the cultural interaction and experience of intangible cultural heritage, but also arouse more people's interests in cultural consumption, especially young people. The accumulation of capital will be achieved through consumption, which will in turn facilitate the financial support for the inheritance of intangible cultural heritage. In this way, the cultural value of intangible cultural heritage can be fully demonstrated, and the social and economic value of it can be further enhanced.

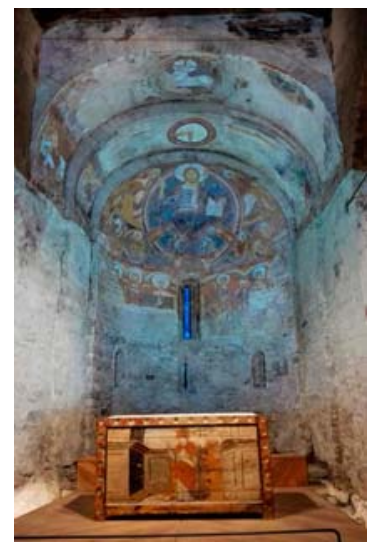

Figure. 2 Author: Francesc Fusté-Forné 


\subsection{Information Processing of Intangible Cultural Heritage Based on Big Data}

Today, the main way of digital informatization of intangible cultural heritage protection in China focuses on the high-speed storage and display of big data, and document visualization has become the most important element in constructing the protection system of intangible cultural heritage. Cloud storage mode of big data is featured by enormous data-carry capacity, clear classification and fast processing speed, which enable it to improve the efficiency and ability of intangible cultural heritage protection. Since it is unable to completely reproduce the objective situation when intangible cultural heritage was flourishing, so after the intangible cultural heritage information is processed and analyzed by using big data technology, an appropriate resource database is constructed, integrated and utilized, which can better meet the requirements of the times, realize the organic connection and integration of ancient and modern information, so as to better offer new opportunities, conditions and means for the inheritance and development of intangible cultural heritage [8]. The storage of big data not only significantly lowers the transmission cost of intangible cultural heritage information and makes the information easy to retrieve, but also greatly improves the safety and stability of data storage.

\section{Emotional Drain In Digitizing CuLtURAL HeRITAGe AND ITS PROTECTION}

\subsection{Emotion in Cultural Heritage}

The emotion of cultural heritage mainly consists of emotional factors which are a representation of humanistic feelings in intangible cultural heritage. Some intangible cultural heritage is not only special in decorative patterns and unique crafts, but also in sensory experience such as smell, sound, touch and even temperature. These emotional factors are often involved in the processes like "scene restoration", "visual sense", "graphic introduction" and "skill experience" in which all these details constitute the important elements of emotional factors (Figure 3).

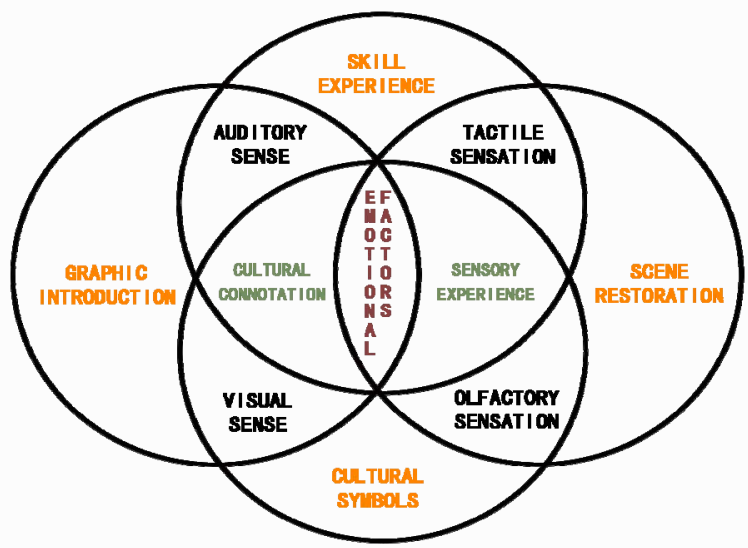

Figure 3. Composition of Emotional Factors

\subsection{Emotional Drain}

\subsubsection{Value the Superficial Expression and Deviate from the Essential Connotations}

The extensive application of digitization may put the protection of intangible cultural heritage on the road of "technology-oriented", thus unconsciously filtering out the cultural connotations. Some digital display technologies used to reproduce intangible cultural heritage may enable people to pay attention to the representational form and unique composition of intangible cultural heritage so that the inheritance and spread of intangible cultural heritage would become only superficial and its vivid, detailed and rich cultural connotations would be ignored. If so, the social value and cultural attributes of intangible cultural heritage can hardly be fully reflected. Some companies have no bottom line in attracting public attention simply in order to maximize their economic benefits. For instance, the Northeast Er-ren-zhuan (a performing form of duet in Northeast China), as intangible cultural heritage, own its artistic value to its unique singing style and real-life plot. But some entertainment companies deliberately modify the scripts and add lots of vulgar contents to attract the audience's attention in the form of VR in order to increase their ticket proceeds. This not only deliberately distorts the connotations and forms of intangible cultural heritage, but also intentionally amplifies some of the dross in intangible cultural heritage that has been deviated from the main theme of the era. In doing this, such practices attempt to replace the real cultural spread with sensory stimulation and all-rounded entertainment, which totally go against the purpose of intangible cultural heritage protection. To some extent, digital display is a virtual space constructed by computer, which has no physical characteristics and has its limitations. Consequently, it is not easy to retain the core connotations of intangible cultural heritage. And designers should have a correct cultural understanding and value judgment of intangible cultural heritage before constructing digital virtual forms of intangible cultural heritage.

\subsubsection{Loss of Emotional Factors in the Spreading Process}

If the emotional factors of intangible cultural heritage products are retained, the disadvantages of traditional digital design that overemphasizes technological expression can be overcome, and so the organic fusion of rational and emotional designs can be realized, and the distance among people, between people and products can be effectively shortened [9]. In light of the current level of technology, it is even hard to present these multi-dimensional sensory experiences through digital technology, let alone direct recording and transmitting. For example, if you want to record the making of Nanan Gambling Mooncake, a kind of food as intangible cultural heritage, you need to know the taste before and after processing, as well as the consistency of the dough 
to ensure deliciousness. Or, if you want to record the sacrificial activities in the gathering area inhabited by the Hui nationality in Southern Fujian, you need to hear the plain instrumental performance by the sacrificial band and perceive people's emotion while feeling the flame temperature. And these are difficult to restore and convey through digital technology. But without these experiences, part of the contents conveyed in intangible cultural heritage and appeal of cultural connotations will be greatly reduced. Objectively speaking, the digital presentation of intangible cultural heritage is actually a process of secondary creation. The reason is that after the designers understand intangible cultural heritage through the materials, they would present the intangible cultural heritage in a digital way according to their own subjective feelings and experiences. But in line with their cultural background and design methods, their representation of the original appearance of the intangible cultural heritage would more or less differ from the real one and part of emotion would be lost (Figure 4).

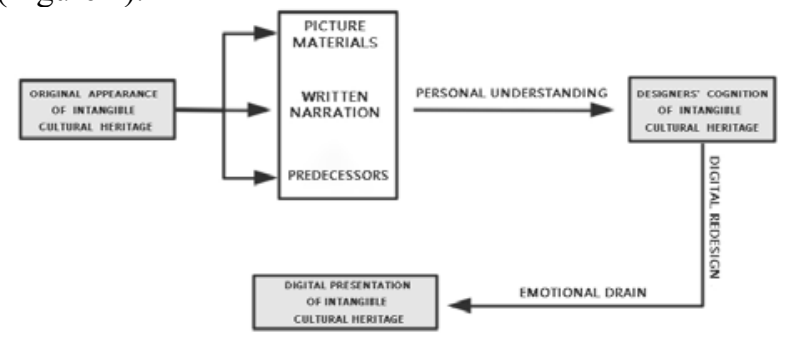

Figure 4. Digital Design Flow of Intangible Cultural Heritage

\subsection{Emotional Protection}

Since emotional factors have become the most important part in the increasingly digitized design research nowadays [10], when intangible cultural heritage is dominated by impersonal technology, we should value the objective record of "emotional factors" before constructing the digital resources of intangible cultural heritage closely related to special sensory experience, so as to make up for some deficiencies. The core lies in that designers should objectively analyze the emotional resonance and extract the core elements from intangible cultural heritage, and then reorganize, optimize and transform these elements. After retaining the recognizable symbols, they should reproduce or express the key skills, and further combine these skills with modern technology.

There is a necessity for us to reflect on the technological advantages of the digitization of intangible cultural heritage, and in the meanwhile respect the "emotional temperature" in the spread of intangible cultural heritage. In addition, we also need to guide the digitization of intangible cultural heritage to shift its focus from cultural attraction to cultural appeal. To make sure that both the spirit and form of intangible cultural heritage can be preserved, inherited and spread in the digitizing process, a slight of "emotional temperature" should be given to rational technology.

\section{CONCLUSION}

In today's fast-developing society, the cultural heritage dominated by new technology is becoming popular, and advocating design research and using new technology has become the mainstream. While exploring the "new world", the charm of traditional culture has been gradually lost in the design process without noticing it. Intangible cultural heritage has survived the passage and hardship of history, indicating that it must possesses visual aesthetic, artistic connotation and cultural value. Its charm lies in the national spirits and and emotional temperature that it conveys. This method can not only play a fundamental role in the spread of intangible cultural heritage, but also retain the "emotional temperature", so as to better transform the intangible cultural assets into the "tangible" spiritual wealth.

\section{REFERENCES}

1. Wei Weiyi. Intangible Cultural Heritage and Regional Development --Taking the Shadow Play of Huaxian County as the Example. 2020, 4(1)

2. Li Fang. A Study of Digital Development of Intangible Cultural Heritage Based on Mixed Reality Technology [J]. Science and Technology \& Innovation, 2016 (12): 40.

3. Zheng Sisi, Chu LeYang, Tan Yue \& Chen Weidong. A Study of Redesign and Utilization of Intangible Cultural Heritage Resources [J]. Art Science and Technology, 2019(03): 18-19.

4. Yang Jielin. Researcb on New Media Communication Strategies on Miao Drum Dances in the Xiangxi, Hunan Provice [D]. Chongqing: Southwest University, 2018: 57.

5. Liu Peilin \&Li Bohua. On the Origin, Misunderstanding and Countermeasures of Digital Protection of Traditional Villages [J]. Journal of Capital Normal University (Social Science Edition), 2008 (05).

6. Zhang Bingfu. On the Inheritance and Development of Intangible Cultural Heritage in the Context of Big Data [J]. The Central Plains Culture Research, 2020 (05): 58-65.

7. Huang Hui. On the Design Programming Method of Interactive Products under Emotional Design [J]. Popular Literature and Art, 2020 (14): 106-107. 